\title{
Chronic constriction injury-induced microRNA-146a-5p alleviates neuropathic pain through suppression of IRAK1/TRAF6 signaling pathway
}

Zhiyao Wang ${ }^{1,3+}$, Fan Liu ${ }^{1,2+}$, Min Wei ${ }^{1}$, Yue Qiu', Chao Ma ${ }^{2^{*}}$, Le Shen ${ }^{1 *}$ and Yuguang Huang ${ }^{1 *}$

\begin{abstract}
Background: microRNA-146a-5p (miRNA-146a-5p) is a key molecule in the negative regulation pathway of TLRs and IL-1 receptor (TIR) signaling. Our recent study demonstrated that MyD88-dependent signaling pathway of TIR in the dorsal root ganglion (DRG) and spinal dorsal horn (SDH) plays a role in peripheral nerve injury-induced neuropathic pain. However, it was not clear whether and how miRNA-146a-5p regulates the TIR pathway of DRG and SDH in the development of neuropathic pain.
\end{abstract}

Methods: The sciatic nerve chronic constriction injury $(\mathrm{CCl})$ model of rat was used to induce chronic neuropathic pain. The levels and cellular distribution of miRNA-146a-5p were detected with quantitative real-time PCR (qPCR) and fluorescent in situ hybridization (FISH). The RNA level, protein level, and cellular distribution of IRAK1 and TRAF6 that is targeted by miRNA-146a-5p were detected with $q P C R$, western blot, and immunofluorescent. The painrelated behavioral effect of miRNA-146a-5p was accessed after intrathecal administration. Mechanical stimuli and radiant heat were used to evaluate mechanical allodynia and thermal hyperalgesia.

Results: We found that the level of miRNA-146a-5p significantly increased in L4-L6 DRGs and SDH after CCI surgery; meanwhile, the protein level of IRAK1 and TRAF6 in DRGs was significantly increased after CCI. Intrathecal injection of miR146a-5p agomir or miRNA-146a-5p antagomir regulates miRNA-146a-5p level of L4-L6 DRGs and SDH. We found that intrathecal injection of miR146a-5p agomir can alleviate mechanical and thermal hyperalgesia in CCI rats and reverse the upregulation of IRAK1 and TRAF6 of L4-L6 DRGs and SDH induced by CCI. We furthermore found that intrathecal injection of miRNA-146a-5p antagomir can exacerbate the mechanical and thermal pain-related behavior of CCI rats and meanwhile increase IRAK1 and TRAF6 of L4-L6 DRGs and SDH expression even further.

Conclusions: miRNA-146a-5p of DRG and SDH can modulate the development of CCl-induced neuropathic pain through inhibition of IRAK1 and TRAF6 in the TIR signaling pathway. Hence, miRNA-146a-5p may serve as a potential therapeutic target for neuropathic pain.

Keywords: miRNA-146a-5p, TRAF6, IRAK1, Dorsal root ganglion, Spinal dorsal horn, Neuropathic pain

\footnotetext{
*Correspondence: machao@ibms.cams.cn; pumchshenle@aliyun.com; garybeijing@163.com

'Zhiyao Wang and Fan Liu contributed equally to this work.

${ }^{2}$ Institute of Basic Medical Sciences Chinese Academy of Medical Sciences, Department of Human Anatomy, Histology and Embryology, Neuroscience Center, School of Basic Medicine, Joint Laboratory of Anesthesia and Pain, Peking Union Medical College, No. 5 DongDanSanTiao, DongChengQu, Beijing 100005, China

${ }^{1}$ Department of Anesthesiology, Peking Union Medical College Hospital, Chinese Academy of Medical Sciences, Peking Union Medical College, Beijing 100730, China

Full list of author information is available at the end of the article
}

(c) The Author(s). 2018 Open Access This article is distributed under the terms of the Creative Commons Attribution 4.0 International License (http://creativecommons.org/licenses/by/4.0/), which permits unrestricted use, distribution, and reproduction in any medium, provided you give appropriate credit to the original author(s) and the source, provide a link to the Creative Commons license, and indicate if changes were made. The Creative Commons Public Domain Dedication waiver (http://creativecommons.org/publicdomain/zero/1.0/) applies to the data made available in this article, unless otherwise stated. 


\section{Background}

Neuropathic pain is a rather stubborn pain induced by nerve injury. It can persist for months to years, even after the primary injury has healed [1]. Many studies focus on the molecular mechanisms are related to neuropathic pain. However, there is no medication currently available that treat neuropathic pain in a complete and definitive way. Accumulating evidence demonstrates that neuroinflammation in the peripheral and central nervous system (e.g., dorsal root ganglion (DRG) and spinal dorsal horn $(\mathrm{SDH})$ ) is involved in peripheral nerve injury-induced neuropathic pain [2-4]. DRG neurons are responsible for the complication of neuropathic pain as they include mechanoceptor, thermoceptor, and pruritic sensor [2]. Peripheral nerve injury activates nociceptive pathways and alters gene expression in DRG neurons, which may contribute to the development and maintenance of neuropathic pain.

Recent studies describe immune-related proteins of DRG and SDH are key players for peripheral and central sensitization of neuropathic pain [5-8]. Toll/interleukin-1 receptors (TIRs) such as TLR4 and IL-1R are found not only expressed on immune cells but also on sensory neurons in DRGs and glial cells (microglia and astrocytes) in the SDH [9-14]. Targeting toll-like receptors (TLRs) such as TLR4 expressed on spinal glial cells has been reported to relieve mice neuropathic pain [5]. Our recent studies show that suppression of myeloid differentiation factor-88 adaptor protein (MyD88)-dependent signaling alleviates neuropathic pain induced by peripheral nerve injury in the rat [15]. The MyD88 is involved in TIRs, mediates activation of TIRs, leads to the NF- $\mathrm{kB}$ activation, and induces proinflammatory mediators [9, 16]. TIRs and its signaling pathway play important roles in the pathogenesis of neuropathic pain. The activation of TIRs also needs to recruit interleukin-1 receptor-associated kinase 1 (IRAK1) and tumor necrosis factor receptor-associated factor 6 (TRAF6) to activate NF- $\mathrm{kB}$ signaling pathway [16].

Recent studies found the activation of NF- $\mathrm{kB}$, and binding the promoters NF-kB-sensitive genes induce transcription of hundreds genes including NF- $\mathrm{kB}$-dependent miRNAs such as miRNA146a-5p [17, 18]. miRNA is a family of small endogenous non-coding RNA molecules that silence target mRNAs by binding to their 3'UTRs. The miRNAs of the DRG participate in nociceptive modulation in the somatosensory pain [19]. miRNAs affect neuropathic pain by regulating key proteins in the pain progress, resulting in hyperalgesia and allodynia [20]. Mounting evidence suggests that miRNA-146a-5p is involved in the innate immune response and can reduce inflammation by targeting both TRAF6 and IRAK1 in monocytes, macrophages, and astrocytes [21-24]. Previous research demonstrated that spinal miRNA-146a could contribute to osteoarthritic pain of knee joints [25]. Also, $\mathrm{Lu}$ et al. found that miRNA-146a of astrocytes could attenuate SNL-induced neuropathic pain by suppressing TRAF6 signaling in the spinal cord [26]. However, the role of miRNA-146a-5p in DRG and SDH of nerve injury-induced neuropathic pain has not been fully investigated. How miRNA-146a-5p modulates the downstream target gene of DRG neurons in chronic constriction injury (CCI) is still unknown. TRAF6 and IRAK1 of TIR signaling may play an important role for neuroinflammation in DRG neurons of CCI model.

In the current study, we evaluated the expression of miRNA-146a-5p and its target genes, namely, IRAK1 and TRAF6, in the DRG of rats with CCI. We also intrathecally administered miRNA-146a-5p agonist (miRNA-146a-5p agomir) or antagonist (miRNA-146a-5p antagomir) to investigate the function of miRNA-146a-5p in modulating neuropathic pain. Our data demonstrated that miRNA-146a-5p can alleviate CCI-induced mechanical and thermal hyperalgesia through inhibition of IRAK1 and TRAF6 and may be the target for protection against chronic pain.

\section{Methods}

Animals

Male Sprague-Dawley (SD) rats weighing 200-250 g were acquired from Laboratory Animal Center of Peking Union Medical College Hospital, Chinese Academy of Medical Sciences. Animals were randomly assigned to treatment or control groups. These rats were bred in a specific pathogen-free environment in 12-h light-dark cycle. The rats were fed with rodent diet and water. These experiments were approved by the Institutional Animal Care and Use Committee in Chinese Academy of Medical Sciences.

\section{Rat model of neuropathic pain}

In accordance with the study of Bennett and Xie YK [27], we performed CCI on rats anesthetized through intraperitoneal injection of sodium pentobarbital $(40 \mathrm{mg} / \mathrm{kg})$ under aseptic condition. After the sciatic nerve of the mid-thigh level on each side was exposed, four snug ligatures of chromic gut suture were loosely tied around the nerve with about $1-\mathrm{mm}$ space between the knots. The sciatic nerves of sham animals were exposed without ligation.

\section{Behavioral test}

Eight rats were included in each group. Paw withdrawal threshold (PWT) in response to mechanical stimuli was used to access mechanical allodynia by using Von Frey filaments 1 day before operation and 1, 3, 5, 7, 14, and 21 days after the operation. Paw withdrawal latency (PWL) in response to radiant heat was used to evaluate thermal hyperalgesia. Three repeat measures were 
performed in each rat with a 5-min interval. This test was performed at 10 a.m. on day 1 preoperation and days $1,3,5,7,14$, and 21 postoperation. At the end of behavior testing, the L4-L6 DRGs and SDH were chronologically harvested and rapidly frozen at $-80^{\circ} \mathrm{C}$.

Intrathecal catheter implantation and intrathecal injection Eight rats were included in each group. A PE10 catheter (length, $15 \mathrm{~cm}$ ) was intrathecally implanted using a previously described technique [28, 29]. Briefly, rats were intraperitoneally anesthetized with $10 \%$ chloral hydrate $(300 \mathrm{mg} / \mathrm{kg})$. A partial laminectomy at L5/L6 was performed to position the intrathecal catheter, and the dural membrane was exposed. The catheter was inserted through a dural incision and passed by $2 \mathrm{~cm}$ into the intrathecal space. The catheter was secured with $4 / 0$ silk threads to the bones and muscles. After implantation, all rats were allowed to recover for a minimum of 2 days prior to the experiments. Rats presenting motor weakness or signs of paresis upon recovery from anesthesia were killed. Proper location of the catheter was confirmed through hind limb paralysis after injection of $10 \mu \mathrm{L}$ of $2 \%$ lidocaine.

Intrathecal drug was administered with a microinjection syringe connected to the intrathecal catheter. CCI rats were randomly divided for intrathecal injecting miRNA-146a-5p agomir (Ribobio, China), agomir control (Ribobio, China), miRNA-146a-5p anatagomir (Ribobio, China), or antagomir control (Ribobio, China). Each drug $(5 \mathrm{nmol})$ was intrathecally administered in $20 \mu \mathrm{L}$ volume on the surgery day and on days 4,8 , and 12 after CCI surgery.

\section{Quantitative real-time PCR}

Total RNA was isolated with TRIzol reagent (Invitrogen Life Technologies) and reverse-transcribed using a reaction mixture in accordance to the manufacturer's instruction. RNA quality and quantity were determined with a NanoDrop spectrophotometer (ND-1000; NanoDrop Technologies), and RNA integrity was assessed through gel electrophoresis. Quantitative real-time PCR (qPCR) was performed on a StepOnePlus real-time PCR system (Applied Biosystems, ABI, CA, USA) using the SYBR Green qPCR Master Mix (ABI, CA, USA). Expression data were normalized to the expression of $\beta$-actin. The total RNA was reverse-transcribed to determine the miRNA expression, and the resulting cDNA was mixed with miRNA-specific Taqman primers (ABI, CA, USA) and Taqman Universal PCR Master Mix (ABI, CA, USA). U6 RNA was used as an endogenous control for data normalization of the miRNA level. These primers used for SYBR Green qPCR are shown in Table 1. Relative changes in expression were measured using the comparative threshold cycle $(\mathrm{Ct})$ method and $2^{-\Delta \Delta \mathrm{Ct}}$ as
Table 1 Primer set list for $\mathrm{qPCR}$

\begin{tabular}{lll}
\hline Target gene & & Primer sequence 5'-3' \\
\hline IRAKI & Forward & GCTCCCAGACCCATTCTGAG \\
& Reverse & CTCTGGGCTGGCTTGATGG \\
TRAF6 & Forward & GCCCATGCCGTATGAAGAGA \\
& Reverse & ACTGAATGTGCAGGGGACTG \\
$\beta$-actin & Forward & CACCCGCGAGTACAACCTTC \\
& Reverse & CCCATACCCACCATCACACC \\
\hline
\end{tabular}

previously described; the results indicated the fold change of expression.

\section{Fluorescent in situ hybridization}

To examine expression of miR-146a in DRG neurons, in situ hybridization was used with locked nucleic acid probes specific for miR-146a. Rats were sacrificed under anesthesia. L4-L6 DRGs were fixed by 4\% paraformaldehyde. After incubated in hybridization solution at room temperature for $2 \mathrm{~h}$, the sections were incubated overnight in hybridization solution with $8 \mathrm{ng} / \mu \mathrm{L}$ of FAM (488) labeled probes for miR-146a-5p (5'-FAM-AACCC ATGGA ATTCA GTTCT CT-FAM-3', Wuhan Servicebio technology) at $37^{\circ} \mathrm{C}$. The sections were washed in $2 \times \mathrm{SSC}$ at $37^{\circ} \mathrm{C}$ for $10 \mathrm{~min}$ and in $0.5 \times \mathrm{SSC}$ at room temperature for $10 \mathrm{~min}$. Slides were then coverslipped with VECTASHIELD Mounting Medium with DAPI.

\section{Immunohistochemistry}

After the rats were anesthetized with sodium pentobarbital, they were perfused transcardially with fresh 4\% paraformaldehyde. L4-L6 DRGs were harvested, postfixed in $4 \%$ paraformaldehyde for $2 \mathrm{~h}$, and then dehydrated in $30 \%$ sucrose overnight at $4{ }^{\circ} \mathrm{C}$. The tissues were embedded in the optimal cutting temperature compound according to our previous studies. Frozen sections (each with $15 \mu \mathrm{m}$ thickness) were used for immunohistochemistry analysis. The tissue sections were incubated with following primary antibodies. Then, tissue sections were incubated with the proper secondary antibodies or Alexa Fluor 594-conjugated isolectin B4 (IB4) (1:100, Invitrogen/ Thermo Fisher Scientific, USA) for $1 \mathrm{~h}$. Slides were then washed in PBS and coverslipped with VECTASHIELD Mounting Medium with DAPI. Table 2 lists the primary and secondary antibodies used for the immunofluorescence staining analysis.

\section{Western blot}

Total proteins from rat L4-L6 DRGs or SDH were extracted with lysis buffer (CWBio, Beijing, China). Briefly, $30 \mu \mathrm{g}$ of each sample was resolved through sodium dodecyl sulfate polyacrylamide gel electrophoresis 
Table $\mathbf{2}$ List of primary and secondary antibodies used for immunofluorescence staining

\begin{tabular}{|c|c|c|c|c|c|}
\hline Antibody & Host & Company & Catalog ID & Dilution & Incubation conditions \\
\hline IRAK1 & Rabbit & Abcam & ab238 & $1: 200$ & Overnight $4{ }^{\circ} \mathrm{C}$ \\
\hline TRAF6 & Rabbit & Abcam & ab181622 & $1: 200$ & Overnight $4{ }^{\circ} \mathrm{C}$ \\
\hline pNF-kB (p65) & Rabbit & Abcam & ab86299 & $1: 200$ & Overnight $4{ }^{\circ} \mathrm{C}$ \\
\hline CGRP & Goat & LifeSpan BioSciences & LS-C122785 & $1: 500$ & Overnight $4{ }^{\circ} \mathrm{C}$ \\
\hline PGP9.5 & Guinea pig & Abcam & ab10410 & 1:100 & Overnight $4{ }^{\circ} \mathrm{C}$ \\
\hline Anti-rabbit IgG Alexa Fluor 488 & Donkey & Jackson ImmunoRresearch & $711-545-152$ & 1:400 & $1 \mathrm{~h} \mathrm{RT}$ \\
\hline Anti-goat IgG Alexa Fluor 594 & Donkey & Jackson ImmunoRresearch & $705-585-147$ & $1: 400$ & $1 \mathrm{~h} \mathrm{RT}$ \\
\hline
\end{tabular}

and then transferred onto Immobilon-P polyvinylidene difluoride (GE). After blocking with 5\% BSA for $1 \mathrm{~h}$ at room temperature, the membranes were incubated with an anti-IRAK1 antibody, anti-TRAF6 antibody, anti-pNF-kB (p65) antibody, and anti- $\beta$-actin antibody. The corresponding secondary antibodies were probed after washing the membranes. Final results were acquired using a western blot detection system (GE) with enhanced chemiluminescence reagents eECL Kit (CWBio, Beijing, China). Table 3 lists the primary and secondary antibodies used for the western blot analysis.

\section{Statistical analysis}

Data are expressed as mean and standard errors (mean \pm SEM). Statistical analyses were performed using SPSS software (vision 17.0). Differences between two groups were analyzed using Student's $t$ test. One-way ANOVA followed by Bonferroni's post hoc tests was used to determine statistical differences among western blot and qPCR. Two-way ANOVA followed by Bonferroni's post hoc tests was used to analyze the behavioral data. $P<0.05$ was considered statistically significant.

\section{Results}

Expression level of miRNA-146a-5p is elevated in DRG and SDH neurons of rat after $\mathrm{CCl}$

The TIR signaling is critical for nerve injury-induced neuropathic pain generation and maintenance. Our recent results revealed that $\mathrm{CCI}$ increased the level of phospho-NF-kappaB of DRG. The miRNA-146a-5p is the microRNA of NF-kappaB-dependent induction. To investigate the role of miRNA-146a-5p in nerve injury-induced neuropathic pain, we used the CCI model of rat to induce neuropathic pain. Compared with sham operation rats, CCI group rats showed a rapid and persistent mechanical allodynia and thermal hyperalgesia which achieved a significant decrease in paw mechanical threshold and thermal withdrawal latency from postoperative day (POD) 3 to POD21 (Fig. 1a-b).

We first examined the expression level of miRNA-146a-5p in the rat DRG of CCI model. The qPCR results showed that miRNA-146a-5p of CCI expression was gradually, slightly, and long-lastingly increased from POD3 to POD14 and recovered to the sham level at POD21 in the DRG of rat (Fig. 1c). We further determined the cellular distribution of miRNA-146a-5p in the DRG of CCI operation with fluorescent in situ hybridization (FISH). We found that the miRNA-146a-5p was distributed in large-sized, medium-sized, and small neurons, but increased staining miRNA-146a-5p was distributed in the DRG of CCI (Fig. 1d-e). In the SDH, our qPCR analysis showed that the CCI operation produced increased from POD7 to PDO21 in the miRNA-146a-5p level (Fig. 1f). FISH results showed that the increased miRNA-146a-5p was distributed in the SDH of the spinal cord (Fig. 1g-h).

\section{$\mathrm{CCl}$ increases level of IRAK1 and TRAF6 in DRG neurons of rat}

IRAK1 is recruited by MyD88 and initiate TIR signaling. Our published results revealed that MyD88 protein was

Table 3 List of primary and secondary antibodies used for western blot analysis

\begin{tabular}{llllll}
\hline Antibody & Host & Company & Catalog number & Dilution & Incubation conditions \\
\hline IRAK1 & Rabbit & Abcam & ab238 & $1: 1000$ & Overnight $4{ }^{\circ} \mathrm{C}$ \\
TRAF6 & Rabbit & Abcam & ab181622 & $1: 1000$ & Overnight $4{ }^{\circ} \mathrm{C}$ \\
pNF-kB (p65) & Mouse & Cell signaling technology & $\# 13346$ & $1: 1000$ & Overnight $4{ }^{\circ} \mathrm{C}$ \\
B-actin & Mouse & ZSGB-BIO & TA-09 & $1: 1000$ & Overnight $4{ }^{\circ} \mathrm{C}$ \\
Anti-rabbit lgG horseradish peroxidase (HRP) & Goat & ZSGB-BIO & ZDR-5306 & $1: 3000$ & $1 \mathrm{~h} \mathrm{RT}$ \\
Anti-mouse IgG horseradish peroxidase (HRP) & Goat & ZSGB-BIO & ZDR-5307 & $1: 3000$ & $1 \mathrm{~h} \mathrm{RT}$ \\
\hline
\end{tabular}



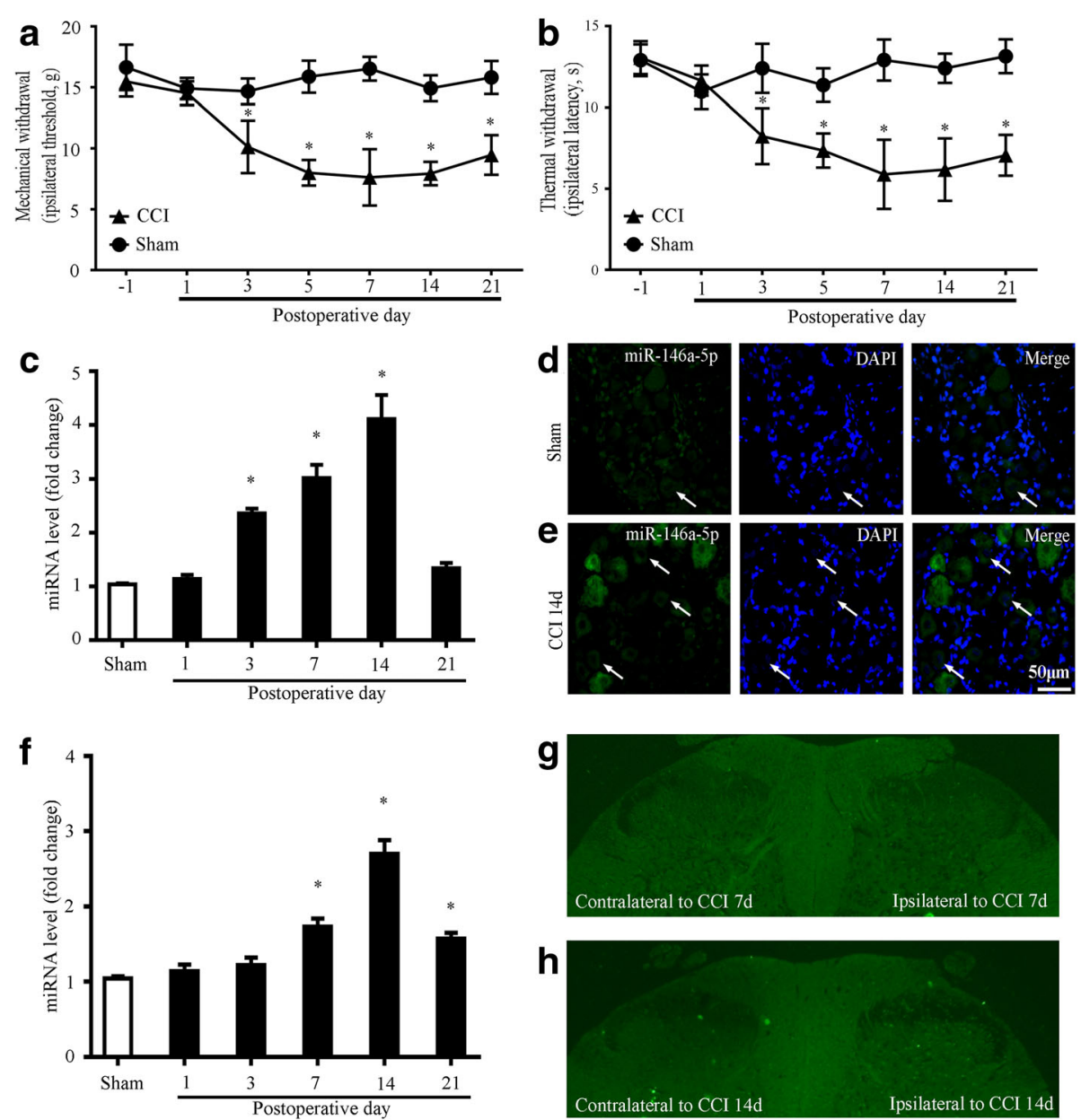

Fig. 1 Expression of miRNA-146a-5p in rat DRG and SDH after CCI. a, b CCl-induced mechanical allodynia and thermal hyperalgesia manifested as a lowered threshold of mechanical withdrawal (a) and thermal withdrawal (b). Eight rats were included in each group. Two-way ANOVA, $* P<0.05$, versus sham. $\mathbf{c}$ qPCR showing the time course for miRNA-146a-5p level in DRG ( $n=4$ in each group). One-way ANOVA, ${ }^{*} P<0.05$, versus sham. $\mathbf{d}$, e FISH showing expression and distribution of miRNA-146a-5p in rat DRGs of sham (d) and CCI 14 days (e). Scale bar $50 \mu$ m. $\mathbf{f}$ qPCR showing the time course for miRNA-146a-5p level in SDH ( $n=4$ in each group). One-way ANOVA, ${ }^{*} P<0.05$, versus sham. $\mathbf{g}, \mathbf{h}$ FISH showing expression and distribution of miRNA-146a-5p in rat SDH of CCl 7 days ( $\mathbf{g})$ and $\mathrm{CCl} 14$ days (h)

increased in the DRG of CCI rats. To examine IRAK1 expression level in the DRG of CCI rats, qPCR results revealed that mRNA level of IRAK1 was continuously increased from POD3 to POD21 in DRG after CCI (Fig. 2a). Similar with the mRNA, western blot analysis showed that the protein expression of IRAK1 was significantly increased in POD7 and peaked in POD14 in DRG after CCI (Fig. 2b). To check the cellular distribution of IRAK1 in the DRG, we did stain of immunofluorescence of IRAK1. Our results showed that IRAK1 immunoreactivity (IRAK1-IR) cells were distributed in the three-size category of DRG neurons of sham and CCI group. We found the percentages of IRAK1-IR neurons of DRG were significantly increased after CCI operation (Fig. 2c-d and Additional file 1: Figure S1).
We also found IRAK1 was expressed in calcitonin gene-related peptide (CGRP)-positive neurons and IB4-positive neurons in the DRG (Fig. 2e-f).

The mRNA level of TRAF6 was also detected through qPCR. We found the mRNA level of TRAF6 was increased in POD3, peaked in POD14, and continuously increased to POD21 (Fig. 3a). We then examined the protein level of TRAF6 in rat DRG. western blot results showed that the expression of TRAF6 was started increasing in POD3 and maintained in POD21 after CCI operation compared with the sham operation (Fig. 3b).

We also examined the distribution of TRAF6 in DRG. Immunofluorescence results showed that TRAF6 immunoreactivity (TRAF6-IR) cells were distributed in the 
a

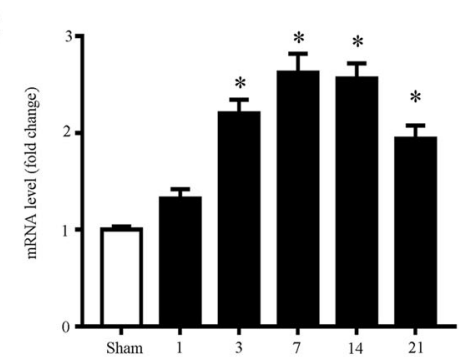

C

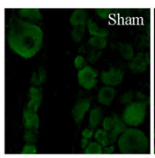

e

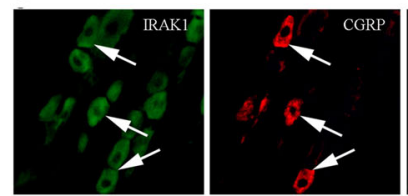

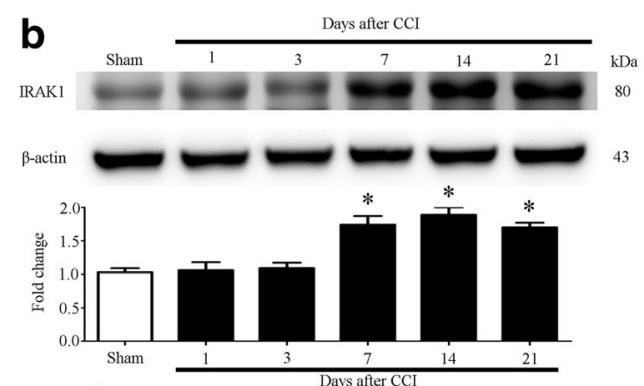

d

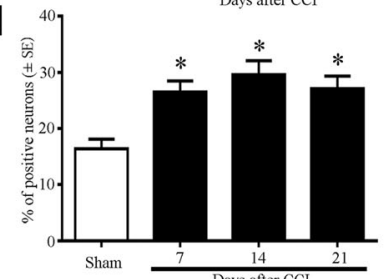

f

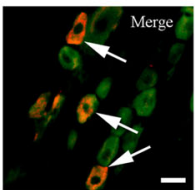

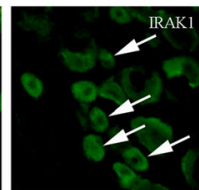

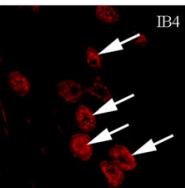

Fig. 2 Expression and cellular distributions of IRAK1 in rat DRG after CCI. a qPCR showing the time course for IRAK1 level in DRG $(n=4$ in each group). One-way ANOVA, ${ }^{*} P<0.05$, versus sham. $\mathbf{b}$ Western blot analyzes the time course for IRAK1 expression in DRG. Representative bands are shown on the top; data summary is shown on the bottom. One-way ANOVA, ${ }^{*} P<0.05$, versus sham. c Immunofluorescence showing cellular distribution of IRAK1 in rat DRGs of sham, CCI 7-day, CCI 14-day, and CCI 21-day group. d Quantification analysis from immunofluorescence staining results of IRAK1 in DRG neurons. Percentage of IRAK1-positive neurons in DRGs of sham, CCI (7 days), CCI (14 days), and CCI (21 days) rats. $n=4$ in each group, One-way ANOVA, ${ }^{*} P<0.05$, versus sham. e Double immunostaining showing co-expression of IRAK1 (green) with CGRP (red) in rat DRG of CCI 14 day. $\mathbf{f}$ Double immunostaining showing co-expression of IRAK1 (green) with IB4 (red) in rat DRG of CCI 14 days. Scale bar $25 \mu \mathrm{m}$ in $\mathbf{c}$, e, and $\mathbf{f}$

three-size category of DRG neurons in the CCI group. CCI induced a marked increase of TRAF6-IR in the ipsilateral side of the DRG at POD7, POD14, and POD21 (Fig. 3c, d and Additional file 1: Figure S2). To check whether TRAF6 is expressed in the nociceptive neuron, we did double immunofluorescence of TRAF6 with nociceptive neuronal markers CGRP and IB4. We found that TRAF6-IR was colocalized with CGRP and IB4 (Fig. 3e, f).

Intrathecal injection miRNA-146a-5p agomir and miRNA146a-5p antagomir regulate miRNA-146a-5p expression levels in DRG and SDH

Agomir is a double-stranded miRNA that is specially marked and chemically modified to regulate the biology of the target gene. To evaluate the effect of miRNA-146a-5p agomir for increasing the miR146a-5p level, we intrathecally injected miRNA-146a-5p agomir in native rats. The expression level of miRNA-146a-5p in SDH and DRG was accessed by qPCR after intrathecally injected miRNA-146a-5p agomir. qPCR results indicated that the expression level of miRNA-146a-5p was increased in L4-L6 DRGs and SDH of rats after intrathecally injected with miRNA-146a-5p agomir compared with agomir control rats (Fig. 4a, b). We also evaluated the effect of miRNA-146a-5p antagomir on the expression of miR-146a-5p and found that compared to the antagomir control group, miRNA-146a-5p antagomir decreased the expression level of miR-146a-5p (Fig. 4c, d).

\section{miRNA-146a-5p agomir relieves $\mathrm{CCl}$-induced neuropathic} pain

Compared with CCI rats that were injected intrathecally with agomir control, injection intrathecally with miRNA-146a-5p agomir significantly attenuated CCI-induced neuropathic pain from POD7 until POD21 (Fig. 5a, b). Subsequently, our qPCR results found that injection intrathecally with miRNA-146a-5p agomir significantly decreased the IRAK1 mRNA level in SDH and DRG of rats with CCI POD14 (Fig. 5c, d). In addition, we also found that the TRAF6 mRNA level in SDH and DRG were decreased in CCI rats with intrathecal miRNA-146a-5p agomir (Fig. 5e, f). We further investigated the potential effects of miRNA-146a-5p agomir on 
a

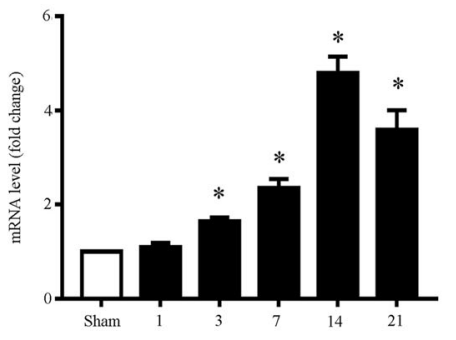

C

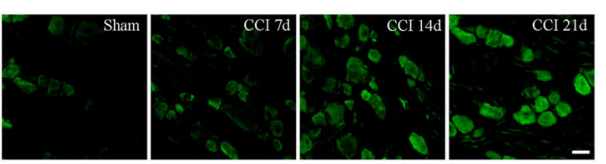

e

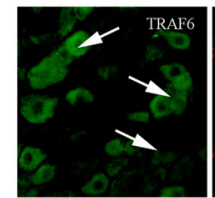

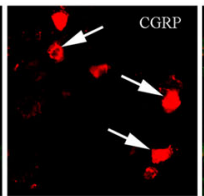

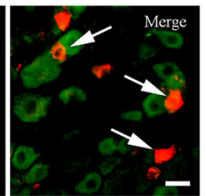

b

d
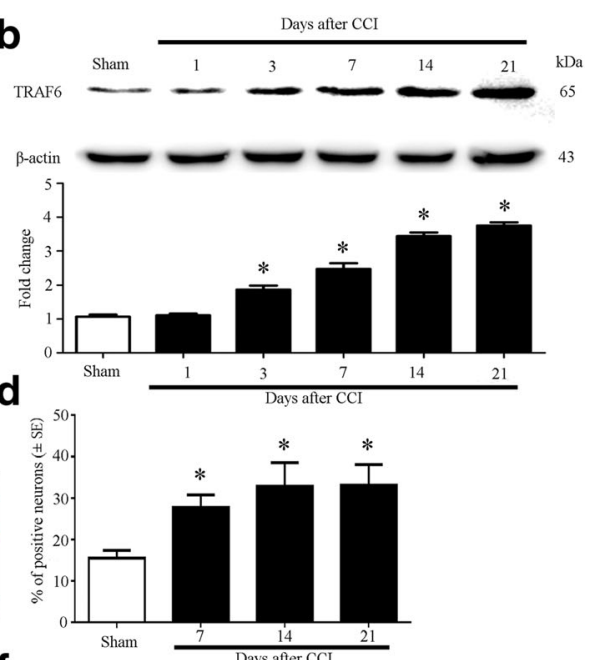

f
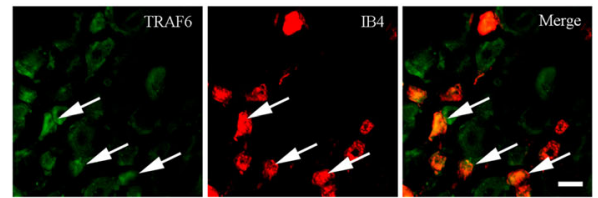

Fig. 3 Expression and cellular distributions of TRAF6 in rat DRG after CCI. a qPCR showing the time course for TRAF6 level in DRG ( $n=4$ in each group). One-way ANOVA, ${ }^{*} P<0.05$, versus sham. $\mathbf{b}$ Western blot analyzes the time course for TRAF6 expression in DRG. c Cellular distribution of TRAF6 in rat DRGs of sham, CCI 7 days, CCl 14 days, and CCl 21 day. $\mathbf{d}$ Percentage of TRAF6-positive neurons in DRGs of sham, CCl (7 days), CCl (14 days), and CCI (21 days) rats. $n=4$ in each group, One-way ANOVA, ${ }^{*} P<0.05$, versus sham. e Double immunostaining showing co-expression of TRAF6 (green) with CGRP (red) in rat DRG of CCI 14 day. $\mathbf{f}$ Double immunostaining showing co-expression of TRAF6 (green) with IB4 (red) in rat DRG of $\mathrm{CCl} 14$ days. Scale bar $25 \mu \mathrm{m}$ in $\mathbf{c}$, e, and $\mathbf{f}$

IRAK1 and TRAF6 protein level in DRG and SDH at 14 days after CCI. In comparison with the rats in the CCI rats injected with negative control, western blot showed that miRNA-146a-5p agomir significantly decreased the protein level of IRAK1 and TRAF6 in DRG and SDH (Fig. 5g, h). Meanwhile, our western blot results also found that intrathecal miRNA-146a-5p agomir decreased the phosphorylation level of $\mathrm{pNF}-\mathrm{kB}$ (p65) protein in CCI rats (Fig. $5 \mathrm{~g}, \mathrm{~h}$ ). Our double immunofluorescence results showed $\mathrm{CCI}$ induced nuclear translocation of pNF-kB (p65) in DRG neurons of rats which were intrathecally injected with miRNA-146a-5p agomir or control agomir (Fig. 5i, j).

miRNA-146a-5p antagomir aggravate neuropathic pain of rats after $\mathrm{CCl}$

We further determined the effect of miR-146a-5p antagomir on CCI rats. Compared with CCI rats that were injected intrathecally antagomir control, injection intrathecally with miR-146a-5p antagomir significantly aggravated CCI-induced neuropathic pain from POD7 to POD21 (Fig. 6a, b). Our qPCR results suggested that
miR-146a-5p antagomir increased the mRNA level of IRAK1 and TRAF6 in DRG and SDH at 14 days after CCI compared with CCI rats which were intrathecally injected with antagomir control (Fig. 6c, f). Western blot results showed that miR-146a-5p antagomir increased the protein level of IRAK1 and TRAF6 in DRG and SDH at CCI POD14 (Fig. 6g-j). We also found intrathecal miR-146a-5p antagomir increased the phosphorylation level of pNF- $\mathrm{kB}$ (p65) protein in CCI rats (Fig. $6 \mathrm{~g}-\mathrm{j})$.

\section{Discussion}

Neuropathic pain is a common type of chronic pain that affects the life quality of patients. The exact molecular mechanism of neuropathic pain has not been fully elucidated. Several rat models with partial injury to peripheral nerves have been used to investigate the possible mechanisms. CCI is a commonly used model to mimic the pathophysiological progress of chronic neuropathic pain. In this study, the role of miRNA-146a-5p in the pathophysiological mechanism of neuropathic pain was investigated. Our research successfully established a CCI rat model and found that the mechanical PWT and thermal PWL in the CCI group were significantly lower than 

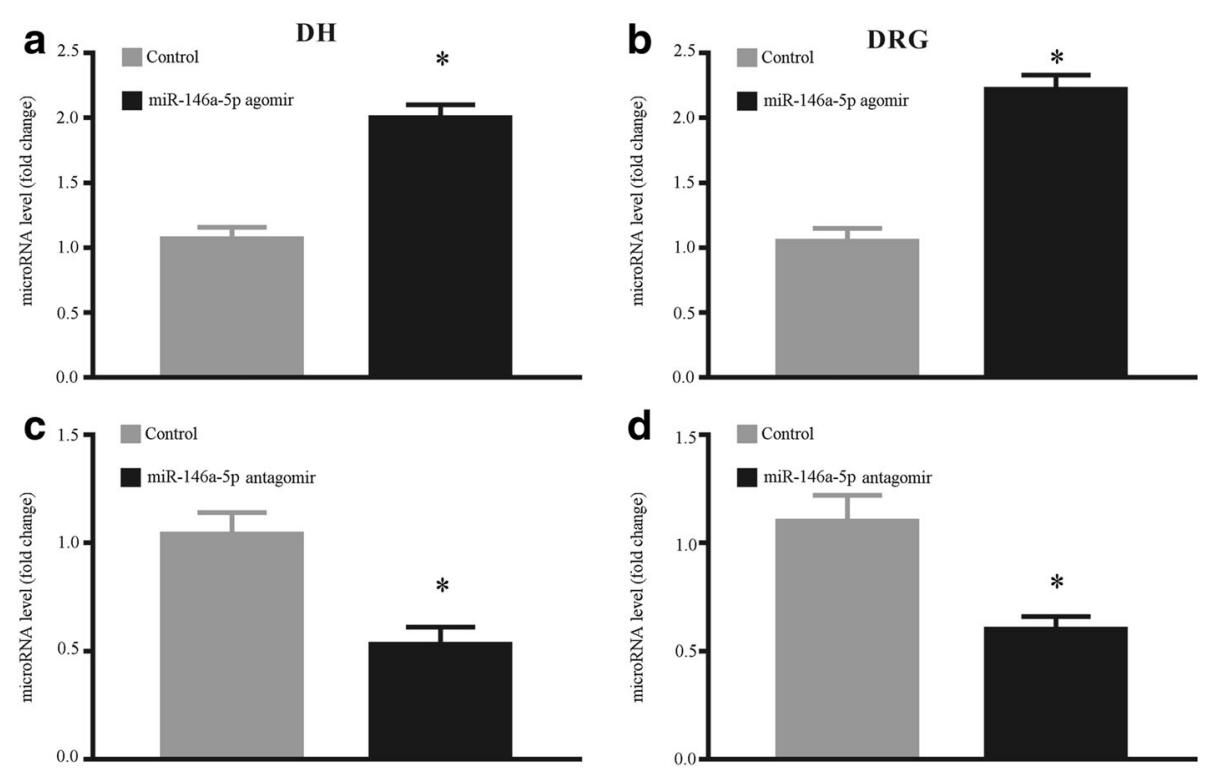

Fig. 4 Expression of miRNA-146a-5p in SDH and DRG of rat with intrathecal administration of miRNA-146a-5p agomir or miRNA-146a-5p antagomir. a b qPCR showed intrathecal administration of miRNA-146a-5p agomir upregulated the RNA level of miRNA-146a-5p in SDH (a) and DRG (b). c, d qPCR showed intrathecal administration of miRNA-146a-5p antagomir reduced the RNA level of miRNA-146a-5p in SDH (c) and DRG (d)

those in the sham group. The CCI rats showed allodynia and hyperalgesia, which are precise clinical characteristics of neuropathic pain. Our results demonstrated a significant increase in the miRNA-146a-5p level in DRG and SDH of rats suffering from neuropathic pain and a considerable increase in the expression of IRAK1 and TRAF6. Our findings are consistent with other studies that used other pain models, in which miRNA-146a-5p, TRAF6, or IRAK1 are strongly upregulated in the SDH of pain models $[26,30,31]$.

Several reports showed some miRNAs participate in the development of neuropathic pain and affect neuropathic pain by regulating protein level in the pain progress [19, 32-34]. The proposed possible mechanism indicates that peripheral stimuli from inflammation or nerve injury can induce the secretion of inflammatory mediators and thus change the miRNA expression in DRG or SDH. miRNA-146a-5p, a member of the miRNA family, is involved in immune responses, cell proliferation, and inflammation [18, 35]. miRNA-146a-5p is related to pain-related pathophysiology of osteoarthritis. The variable expression of miRNA-146a-5p in the spinal cord and DRG contributes to osteoarthritic pain in the knee joint $[25,31]$.

As a critical innate immune receptor, TLRs is activated in neuropathic pain, and its deficiency protects against neuropathic pain. The activation of the TLRs signaling on cells in the peripheral or central nervous system, particularly the glia cell and DRG neuron, contributes to neuropathic pain [5-8, 15, 36, 37]. Activated TLR4 initiates transmembrane signaling cascades that trigger intracellular mediators [13-16]. In this pathway, the activation of IRAK1 and TRAF6 leads to the nuclear translocation of the transcription factor NF- $\mathrm{kB}$, resulting in the production of proinflammatory cytokines, such as IL- 6 and TNF- $\alpha$. Meanwhile, the activation of NF- $\mathrm{kB}$ can induce miRNA-146a-5p [17]. miRNA-146a-5p that is NF- $\mathrm{kB}$-dependent microRNA plays a key role in the regulation of TIR signaling through its target molecules, namely, TRAF6 and IRAK1, which are two important protein kinase in the TIR signaling pathway [17, 22, 24]. We demonstrated that over-expression of miRNA-146a-5p protects rats against neuropathic pain after $\mathrm{CCI}$ operation by negatively regulating the expression level of IRAK1 and TRAF6.

To further determine the role of miRNA-146a-5p in the CCI-induced neuropathic pain, we found CCI rats which were intrathecally injected with miRNA-146a-5p antagonist; miRNA-146a-5p antagomir suffer from aggravated neuropathic pain. Intrathecal injection with miRNA-146a-5p antagomir elevated the level of IRAK1 and TRAF6 of CCI rats. Our finding is consistent with recent studies, in which miRNA-146a-5p negatively regulates the TIR signaling pathway by targeting IRAK1 and TRAF6. Several studies suggested that miRNA-146a-5p may negatively regulate the LPS-induced TLR signaling through downregulation of IRAK1 and TRAF6 by binding to the 3'UTR of their 


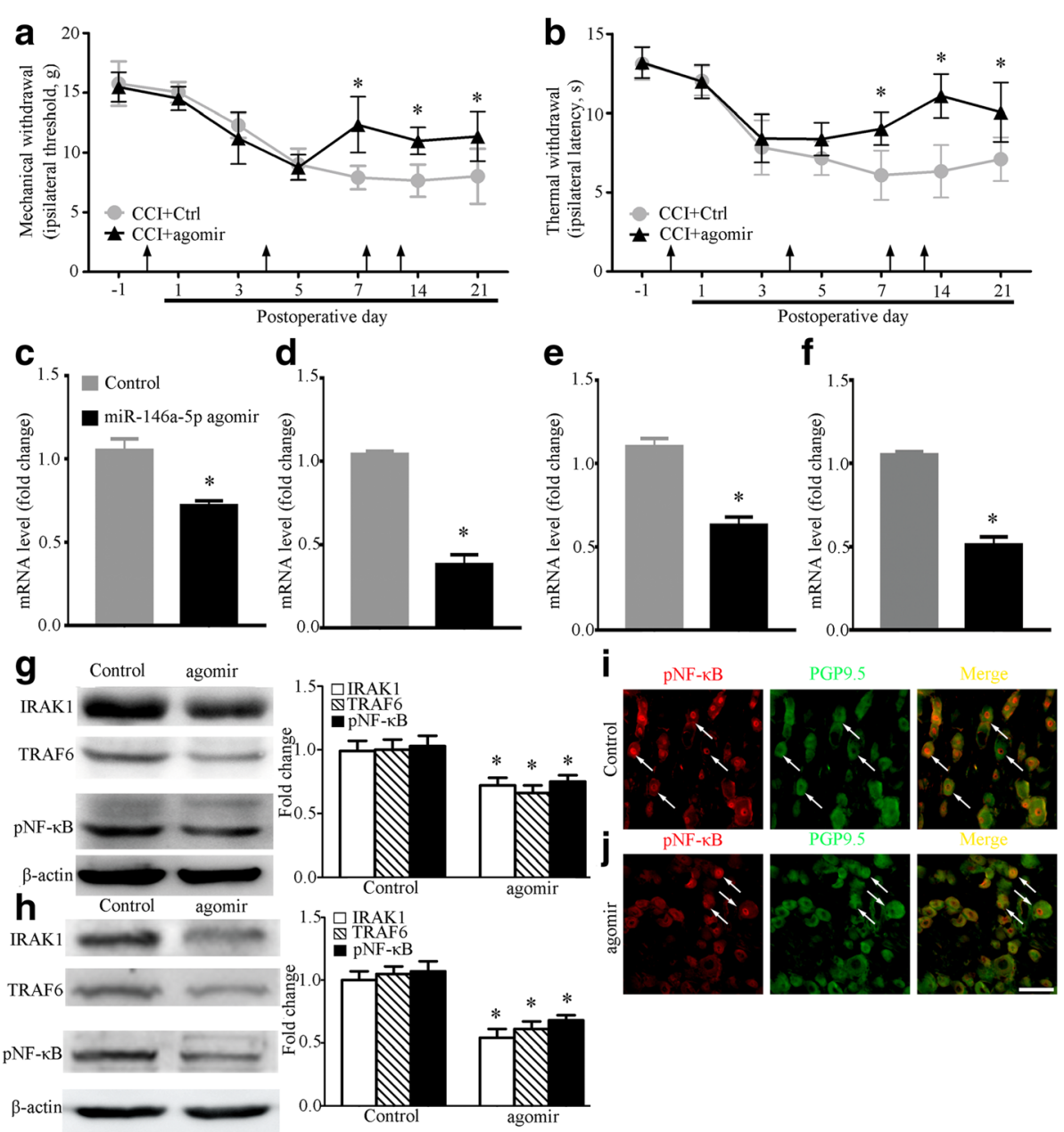

Fig. 5 miRNA-146a-5p attenuated neuropathic pain and decreased IRAK1 and TRAF6 expression in rat DRG and SDH after CCI. a, b Intrathecal injection of miRNA-146a-5p agomir attenuated CCl-induced mechanical allodynia (a) and thermal hyperalgesia (b). Each administration is indicated by an arrow on 0, 4, 8, and 12 days after CCl operation. Two-way ANOVA, ${ }^{*} P<0.05$ versus CCl + Ctrl. miRNA-146a-5p agomir was administrated i.t. in a volume of $20 \mu \mathrm{L}$. The negative miRNA agomir was used in the control group. Eight rats were included in each group. $\mathbf{c}$, $\mathbf{d}$ qPCR showing mRNA level of IRAK1 mRNA in SDH (c) and DRG (d) after miRNA-146a-5p agomir administration. e, $\mathbf{f}$ qPCR showing mRNA level of TRAF6 in SDH (e) and DRG (f) after miRNA-146a-5p agomir administration. $n=4$ in each group of $\mathbf{c}$, $\mathbf{f}$, Student's $t$ test, ${ }^{*} P<0.05$, versus control. $\mathbf{g}$, $\mathbf{h}$ Western blot showing protein level of IRAK1, TRAF6, and pNF-KB (p65) in SDH (g) and DRG (h) after miRNA-146a-5p agomir administration. Data summary is shown on the right. $n=4$ in each group, Student's $t$ test, ${ }^{*} P<0.05$ versus sham. $\mathbf{i}, \mathbf{j}$ Immunofluorescence showing nuclear translocation of pNF-kB (p65) in CCl control group (i) and CCl agomir group (j). Scale bar $50 \mu \mathrm{m}(\mathbf{i}, \mathbf{j})$

mRNAs [17, 38]. Previous studies also confirmed that miRNA-146a-5p-deficient mice exhibit a considerable increase in IRAK1 and TRAF6 protein level and are hypersensitive to LPS [23, 39]. However, whether miR-146a-null mice are sensitive to neuropathic pain must be further confirmed. In our research, we demonstrated that miRNA-146a-5p antagomir increased the phosphorylation level of NF- $k B$ (p65). That indicated downregulation of miRNA-146a-5p may result in the over-responsiveness of TIR signaling pathway. By contrast, the over-expression of miRNA-146a-5p contributed to the lower level of phosphorylation for NF- $\mathrm{kB}$ (p65). In this study, we did not study the expression of miRNA-146a-5p and its targets in the brain after neuropathic pain. The expression of miRNA-146a-5p on the brain can possibly regulate neuropathic pain, yet this hypothesis must be further confirmed.

\section{Conclusions}

In this study, we demonstrated that neuropathic pain was associated with miRNA-146a-5p. The therapeutic approaches using miRNA-146a-5p agomir could relieve neuropathic pain in rat models of CCI. The mechanism may involve the regulation of the TIR signaling pathway 

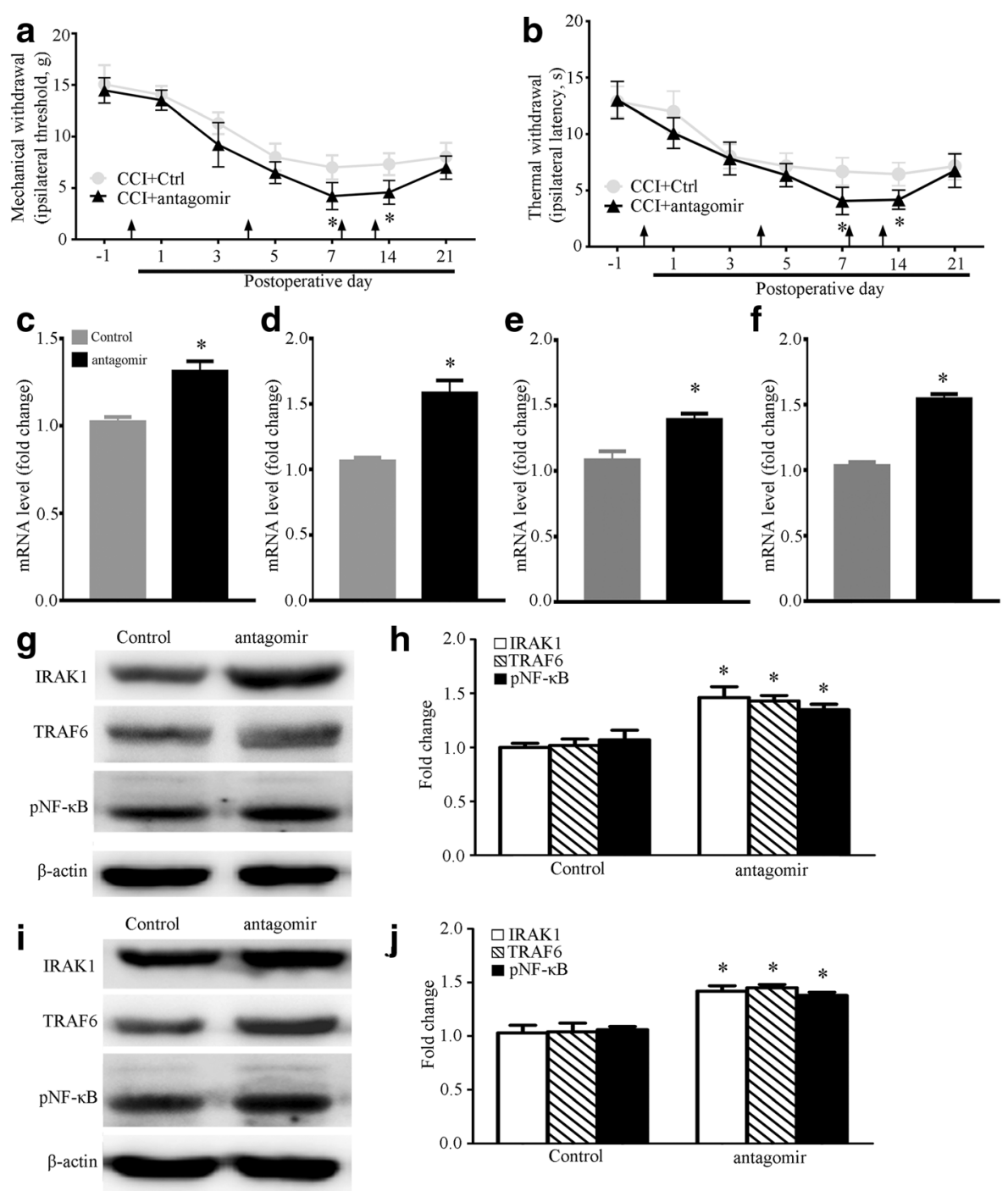

Fig. 6 Inhibition of miRNA-146a-5p leads to aggravated neuropathic pain and increased IRAK1 and TRAF6 expression in rat DRG and SDH after $\mathrm{CCl}$. a, $\mathbf{b}$ Intrathecal injection of miRNA-146a-5p antagomir aggravated CCl-induced mechanical allodynia (a) and thermal hyperalgesia (b). Each administration is indicated by an arrow on $0,4,8$, and 12 days after $C C l$ operation. Two-way ANOVA, ${ }^{*} P<0.05$, versus CCI $+C$ trl. miRNA-146a-5p antagomir was administrated i.t. in a volume of $20 \mu \mathrm{L}$. The negative miRNA antagomir was used in the control group. Eight rats were included in each group. $\mathbf{c}$, $\mathbf{d}$ qPCR showing mRNA level of IRAK1 mRNA in SDH (c) and DRG (d) after miRNA-146a-5p antagomir administration. $\mathbf{e}, \mathbf{f}$ qPCR showing mRNA level of TRAF6 in SDH (e) and DRG (f) after miRNA-146a-5p antagomir administration. $n=4$ in each group of $\mathbf{c}-\mathbf{f}$, Student's $t$ test, ${ }^{*} P<0.05$, versus control. $\mathbf{g}$-j Western blot showing protein level of IRAK1, TRAF6, and pNF-KB (p65) in SDH ( $\left.\mathbf{g}, \mathbf{h}\right)$ and DRG (i, $\left.\mathbf{j}\right)$ after miRNA-146a$5 p$ antagomir administration. Data summary of western blot results in SDH (h) and DRG (j) is shown on the right. $n=4$ in each group, Student's $t$ test, ${ }^{*} P<0.05$ versus sham

by directly suppressing its target, IRAK1 and TRAF6. The administration of miRNA-146a-5p or its inducers can be used as a promising therapy to relieve neuropathic pain.

\section{Additional file}

Additional file 1: Figure S1. Cellular distribution of IRAK1 in DRGs. Figure S2 Cellular distribution of TRAF6 in DRGs. (DOC $925 \mathrm{~kb}$ )

\section{Abbreviations}

CCl: Chronic constriction injury; CGRP: Calcitonin gene-related peptide; DRG: Dorsal root ganglion; FISH: Fluorescent in situ hybridization; IB4: Isolectin B4; IRAK1: Interleukin-1 receptor-associated kinase 1; MyD88: Myeloid differentiation primary response protein 88; NFKB: Transcription factors of the nuclear factor kappa B; POD: Postoperative day; PWL: Paw withdrawal latency; PWT: Paw withdrawal threshold; SDH: Spinal dorsal horn; TIR: Toll/interleukin-1 receptor; TLR: Toll-like receptor; TRAF6: TNF receptor-associated factors 6

\section{Acknowledgements}

We thank Dr. Wenyin Qiu, Xiaojin Qian, and Yongmei Chen in the Department of Anatomy, Histology and Embryology, Institute of Basic 
Medical Sciences Chinese Academy of Medical Sciences, for their technical assistance in immunohistochemistry.

\section{Availability of data materials}

There is no data, software, databases, and application/tool available apart from the reported in the present study. All data is provided in the manuscript and supplementary data.

\section{Funding}

This work was supported by grants from National Science Foundation of China (\#31070930 (Y.H.) and \#81271239, \#81771205, \#91632113 (C.M.)), the PUMC Innovation Fund for Postgraduate (\#2016-1001-09 (F.L.)), the Natural Science Foundation and Major Basic Research Program of Shanghai (16JC1420500, 16JC1420502), and the CAMS Innovation Fund for Medical Sciences (CIFMS \#2017-12M-3-008).

\section{Authors' contributions}

$Z W$ and FL drafted the manuscript. FL performed the immunofluorescence staining. ZW performed the $\mathrm{CCl}$ and behavioral assessment of pain. ZW, MW and $Y Q$ performed the western blot, $\mathrm{QPCR}$, and analysis. ZW, MW, and YQ performed the intrathecal catheterization and drug delivery. FL, CM, LS, and $\mathrm{YH}$ conceived of the study and participated in its design and coordination to draft the manuscript. All authors read and approved the final manuscript.

\section{Ethics approval}

All animal procedures performed in this study were reviewed and approved by the Institutional Animal Care and Use Committee in Chinese Academy of Medical Sciences, Peking Union Medical College Hospital (Beijing, China), and were conducted in accordance with the guidelines of the International Association for the Study of Pain.

\section{Competing interests}

The authors declare that they have no competing interests.

\section{Publisher's Note}

Springer Nature remains neutral with regard to jurisdictional claims in published maps and institutional affiliations.

\section{Author details}

${ }^{1}$ Department of Anesthesiology, Peking Union Medical College Hospital, Chinese Academy of Medical Sciences, Peking Union Medical College, Beijing 100730, China. ${ }^{2}$ Institute of Basic Medical Sciences Chinese Academy of Medical Sciences, Department of Human Anatomy, Histology and Embryology, Neuroscience Center, School of Basic Medicine, Joint Laboratory of Anesthesia and Pain, Peking Union Medical College, No. 5 DongDanSanTiao, DongChengQu, Beijing 100005, China. ${ }^{3}$ Department of Anesthesiology, Zhongshan Hospital, Fudan University, Shanghai 200032, China.

\section{Received: 31 January 2018 Accepted: 24 May 2018}

\section{Published online: 09 June 2018}

\section{References}

1. Haanpaa M, Attal N, Backonja M, Baron R, Bennett M, Bouhassira D, Cruccu G, Hansson P, Haythornthwaite JA, lannetti GD, et al. NeuPSIG guidelines on neuropathic pain assessment. Pain. 2011;152(1):14-27.

2. Colloca L, Ludman T, Bouhassira D, Baron R, Dickenson AH, Yarnitsky D, Freeman R, Truini A, Attal N, Finnerup NB, et al. Neuropathic pain. Nat Rev Dis Primers. 2017:3:17002

3. Finnerup NB, Haroutounian S, Kamerman P, Baron R, Bennett DLH, Bouhassira D, Cruccu G, Freeman R, Hansson P, Nurmikko T, et al. Neuropathic pain: an updated grading system for research and clinical practice. Pain. 2016;157(8):1599-606

4. Woolf CJ. Central sensitization: implications for the diagnosis and treatment of pain. Pain. 2011;152(3 Suppl):S2-15.

5. Bettoni I, Comelli F, Rossini C, Granucci F, Giagnoni G, Peri F, Costa B. Glia TLR4 receptor as new target to treat neuropathic pain: efficacy of a new receptor antagonist in a model of peripheral nerve injury in mice. Glia. 2008;56(12):1312-9.

6. Agalave NM, Larsson M, Abdelmoaty S, Su J, Baharpoor A, Lundback P, Palmblad K, Andersson U, Harris H, Svensson Cl. Spinal HMGB1 induces TLR4- mediated long-lasting hypersensitivity and glial activation and regulates painlike behavior in experimental arthritis. Pain. 2014;155(9):1802-13.

7. Li Y, Zhang H, Zhang H, Kosturakis AK, Jawad AB, Dougherty PM. Toll-like receptor 4 signaling contributes to paclitaxel-induced peripheral neuropathy. J Pain. 2014;15(7):712-25.

8. Lim H, Kim D, Lee SJ. Toll-like receptor 2 mediates peripheral nerve injuryinduced NADPH oxidase 2 expression in spinal cord microglia. J Biol Chem. 2013;288(11):7572-9.

9. Peirs C, Seal RP. Targeting toll-like receptors to treat chronic pain. Nat Med. 2015:21(11):1251-2.

10. Liu T, Han Q, Chen G, Huang Y, Zhao LX, Berta T, Gao YJ, Ji RR. Toll-like receptor 4 contributes to chronic itch, alloknesis, and spinal astrocyte activation in male mice. Pain. 2016;157(4):806-17.

11. Okun E, Griffioen KJ, Mattson MP. Toll-like receptor signaling in neural plasticity and disease. Trends Neurosci. 2011;34(5):269-81.

12. Due MR, Piekarz AD, Wilson N, Feldman P, Ripsch MS, Chavez S, Yin H, Khanna R, White FA. Neuroexcitatory effects of morphine-3-glucuronide are dependent on toll-like receptor 4 signaling. J Neuroinflammation. 2012;9:200.

13. Allette $Y M$, Kim Y, Randolph AL, Smith JA, Ripsch MS, White FA. Decoy peptide targeted to toll-IL-1R domain inhibits LPS and TLR4-active metabolite morphine-3 glucuronide sensitization of sensory neurons. Sci Rep. 2017;7(1):3741

14. Diogenes A, Ferraz CC, Akopian AN, Henry MA, Hargreaves KM. LPS sensitizes TRPV1 via activation of TLR4 in trigeminal sensory neurons. J Dent Res. 2011;90(6):759-64.

15. Liu F, Wang Z, Qiu Y, Wei M, Li C, Xie Y, Shen L, Huang Y, Ma C. Suppression of MyD88-dependent signaling alleviates neuropathic pain induced by peripheral nerve injury in the rat. J Neuroinflammation. 2017;14(1):70.

16. O'Neill LA, Bowie AG. The family of five: TIR-domain-containing adaptors in toll-like receptor signalling. Nat Rev Immunol. 2007;7(5):353-64.

17. Taganov KD, Boldin MP, Chang KJ, Baltimore D. NF-kappaB-dependent induction of microRNA miR-146, an inhibitor targeted to signaling proteins of innate immune responses. Proc Natl Acad Sci U S A. 2006; 103(33):12481-6.

18. Ma X, Becker Buscaglia LE, Barker JR, Li Y. MicroRNAs in NF-kappaB signaling. J Mol Cell Biol. 2011;3(3):159-66.

19. Bali KK, Kuner R. Noncoding RNAs: key molecules in understanding and treating pain. Trends Mol Med. 2014;20(8):437-48.

20. Lutz BM, Bekker A, Tao YX. Noncoding RNAs: new players in chronic pain. Anesthesiology. 2014;121(2):409-17.

21. Iyer A, Zurolo E, Prabowo A, Fluiter K, Spliet WG, van Rijen PC, Gorter JA, Aronica E. MicroRNA-146a: a key regulator of astrocyte-mediated inflammatory response. PLoS One. 2012;7(9):e44789.

22. Hou J, Wang P, Lin L, Liu X, Ma F, An H, Wang Z, Ca X. MicroRNA-146a feedback inhibits RIG-I-dependent type I IFN production in macrophages by targeting TRAF6, IRAK1, and IRAK2. J Immunol. 2009;183(3):2150-8.

23. Chassin C, Hempel C, Stockinger S, Dupont A, Kubler JF, Wedemeyer J, Vandewalle A, Hornef MW. MicroRNA-146a-mediated downregulation of IRAK1 protects mouse and human small intestine against ischemia/ reperfusion injury. EMBO Mol Med. 2012;4(12):1308-19.

24. Rusca N, Monticelli S. MiR-146a in immunity and disease. Mol Biol Int. 2011; 2011:437301.

25. Li X, Kroin JS, Kc R, Gibson G, Chen D, Corbett GT, Pahan K, Fayyaz S, Kim JS, van Wijnen AJ, et al. Altered spinal microRNA-146a and the microRNA-183 cluster contribute to osteoarthritic pain in knee joints. J Bone Mineral Res. 2013;28(12):2512-22.

26. Lu Y, Cao D-L, Jiang B-C, Yang T, Gao Y-J. MicroRNA-146a-5p attenuates neuropathic pain via suppressing TRAF6 signaling in the spinal cord. Brain Behav Immun. 2015;49:119-29.

27. Bennett GJ, Xie YK. A peripheral mononeuropathy in rat that produces disorders of pain sensation like those seen in man. Pain. 1988;33(1):87-107.

28. Lin C-R, Amaya F, Barrett L, Wang H, Takada J, Samad TA, Woolf CJ. Prostaglandin E-2 receptor EP4 contributes to inflammatory pain hypersensitivity. J Pharmacol Exp Ther. 2006;319(3):1096-103.

29. Qiu Y, Chen WY, Wang ZY, Liu F, Wei M, Ma C, Huang YG. Simvastatin attenuates neuropathic pain by inhibiting the RhoA/LIMK/cofilin pathway. Neurochem Res. 2016;41(9):2457-69.

30. Wei J, Wang J, Zhou Y, Yan S, Li K, Lin H. MicroRNA-146a contributes to SCI recovery via regulating TRAF6 and IRAK1 expression. Biomed Res Int. 2016; 2016:4013487. 
31. Li X, Gibson G, Kim J-S, Kroin J, Xu S, van Wijnen AJ, Im H-J. MicroRNA-146a is linked to pain-related pathophysiology of osteoarthritis. Gene. 2011; 480(1-2):34-41.

32. Favereaux A, Thoumine O, Bouali-Benazzouz R, Roques V, Papon MA, Salam SA, Drutel G, Leger C, Calas A, Nagy F, et al. Bidirectional integrative regulation of Cav1.2 calcium channel by microRNA miR-103: role in pain. EMBO J. 2011;30(18):3830-41.

33. von Schack D, Agostino MJ, Murray BS, Li Y, Reddy PS, Chen J, Choe SE, Strassle BW, Li C, Bates B, et al. Dynamic changes in the microRNA expression profile reveal multiple regulatory mechanisms in the spinal nerve ligation model of neuropathic pain. PLoS One. 2011;6(3):e17670.

34. Sengupta JN, Pochiraju S, Kannampalli P, Bruckert M, Addya S, Yadav P, Miranda A, Shaker R, Banerjee B. MicroRNA-mediated GABA Aalpha-1 receptor subunit down-regulation in adult spinal cord following neonatal cystitis-induced chronic visceral pain in rats. Pain. 2013;154(1):59-70.

35. Boldin MP, Taganov KD, Rao DS, Yang L, Zhao JL, Kalwani M, Garcia-Flores Y, Luong M, Devrekanli A, Xu J, et al. miR-146a is a significant brake on autoimmunity, myeloproliferation, and cancer in mice. J Exp Med. 2011; 208(6):1189-201.

36. Villalba M, Hott M, Martin C, Aguila B, Valdivia S, Quezada C, Zambrano A, Concha Ml, Otth C. Herpes simplex virus type 1 induces simultaneous activation of toll-like receptors 2 and 4 and expression of the endogenous ligand serum amyloid A in astrocytes. Med Microbiol Immunol. 2012;201(3):371-9.

37. Kim D, Kim MA, Cho I-H, Kim MS, Lee S, Jo E-K, Choi S-Y, Park K, Kim JS, Akira S, et al. A critical role of toll-like receptor 2 in nerve injury-induced spinal cord glial cell activation and pain hypersensitivity. J Biol Chem. 2007; 282(20):14975-83.

38. Bartel DP. MicroRNAs: target recognition and regulatory functions. Cell. 2009;136(2):215-33.

39. O'Neill LA, Sheedy FJ, McCoy CE. MicroRNAs: the fine-tuners of toll-like receptor signalling. Nat Rev Immunol. 2011;11(3):163-75.

\section{Ready to submit your research? Choose BMC and benefit from:}

- fast, convenient online submission

- thorough peer review by experienced researchers in your field

- rapid publication on acceptance

- support for research data, including large and complex data types

- gold Open Access which fosters wider collaboration and increased citations

- maximum visibility for your research: over $100 \mathrm{M}$ website views per year 